\title{
Meaningful Correlation between the donor registration rate and the number of organ donors by region
}

\author{
Younghwan Hwang, Jungsun Kim, Seungrye Jeong, Miyoung Kim, Minyoung Chu, Jeongrim Lee, Insung Moon
}

Korea Organ Donation Agency, Seoul, Korea

Background: The number of registered organ donation candidates serves as the measure of the public perception of organ donation. This study was planned to investigate the correlation between the numbers of registered organ donation candidates and actual organ donors by region.

Methods: Using the data of the National Population Census by the Korean Statistical Information Service (KOSIS) and the annual statistical data of the Korean Network for Organ Sharing (KONOS) from January 2000 to December 2019, this study compared the numbers of residents and registered organ donation candidates of each region. In addition, based on the total number of organ donors in 2019, a comparison was performed on the numbers of actual organ donors pmp by region.

Results: The statistics over the study period were as follows. With a national population of 51,849,861 people and 1,534,145 registered organ donation candidates, the national rate of organ donation registration was $3.0 \%$. In 2019 , the number of organ donors totaled 450 , with 8.7 organ donors pmp. A comparison by region was carried out for special and metropolitan cities (7) and provinces (8) according to the administrative classification system. As a result of the analysis, of the seven special and metropolitan cities, Gwangju Metropolitan City had the lowest organ donation registration rate (2.5\%). The city also recorded the lowest pmp rate of actual organ donors ( 6.2 persons). Of the eight provinces, Gyeongsangbuk-do province showed the lowest organ donation registration rate $(2.1 \%)$ as well as the lowest pmp rate of actual organ donors pmp (7.5 persons). The results of the comparison of the organ donation registration rates and the numbers of actual donors between the male and female population are as follows. The organ donation registration rate was higher in the female population, with $56.7 \%$ for women and $43.3 \%$ for men, while the number of actual donors pmp was higher in the male population, with $33.3 \%$ for women and $66.7 \%$ for men.

Conclusions: It was found that regions with lower organ donation rate also have lower number of actual donors. The organ donation registration rate reflects the public perception of organ donation. While the analysis results suggest that women have a more positive perception of organ donation, the actual number of donors was higher in men than in women. Therefore, it is necessary to review the proportion of women among those who consent to organ donation. In addition, in regions with low organ donation registration rate, policy support from the central and local governments is needed to promote the culture of organ donation. 